\title{
A Sonic Condition Model for Pseudo-Shock in Divergent Ducts*
}

\author{
Takeshi KANDA ${ }^{1) \dagger}$ and Kento HIKOSAKA ${ }^{2), 3)}$ \\ ${ }^{1)}$ Department of Astronautics and Aeronautics, Chubu University, Kasugai, Aichi 487-8501, Japan \\ ${ }^{2)}$ Department of Mechanical Engineering, Chubu University, Kasugai, Aichi 487-8501, Japan \\ ${ }^{3)}$ Presently, Toyohashi Design Co., Ltd., Toyohashi, Aichi 440-0843, Japan
}

\begin{abstract}
A newly constructed model calculates the starting position of the pseudo-shock, or shock train, in the divergent ducts. The model is based on the conservation laws of mass, energy and momentum of the inflow and outflow into/from the pseudo-shock region, and further uses a sonic condition in the core flow of the pseudo-shock. The model adopts an empirical growth rate of the low-velocity region, and the divergent angle of the nozzles becomes a design parameter. The lengths of the pseudo-shocks calculated show reasonable agreement with the experimental results. Pressure distribution with quick increase near the starting position is simulated. The supersonic core flow cross-section becomes smaller than that at the starting position of the pseudo-shock. The pressure at the sonic point rises to 0.7 of that at the duct exit. The length of the quick pressure-increase region becomes longer as the Mach number increases at the starting position.
\end{abstract}

Key Words: Pseudo-shock, Shock Train, Divergent Duct, Conservation, Sonic Condition

\section{Nomenclature}

A: cross-section, parameter

a: parameter, sonic speed

$B$ : parameter

$b$ : parameter

$c_{f}$ : friction coefficient

$F$ : impulse function, force

$f$ : compressibility effect

$h$ : height

$L$ : length

$M$ : Mach number

$\bar{M}$ : convective Mach number

$\dot{m}$ : mass flow rate

$p$ : pressure

$R$ : gas constant

$R e$ : Reynolds number

$S$ : parameter

$T$ : temperature

$u$ : velocity

$\bar{u}$ : convective velocity

$x$ : distance

$\delta$ : shear layer thickness

$\gamma$ : specific-heat ratio

$\mu$ : viscosity

$\rho:$ density

$\theta$ : convergence angle of supersonic core flow

Subscripts

aw: adiabatic wall

$c$ : supersonic core flow

$d v$ : divergent duct

$e$ : end point of pseudo-shock, environment

(C) 2020 The Japan Society for Aeronautical and Space Sciences *Received 23 October 2019; final revision received 6 January 2020; accepted for publication 13 February 2020.

†Corresponding author, kanda-t@isc.chubu.ac.jp

\author{
$f$ : friction \\ $l$ : low-velocity region \\ $M 1$ : sonic point \\ ps: pseudo-shock \\ $t$ : total \\ w: wall \\ $x: x$ direction \\ 0 : incompressible \\ 1: supersonic uniform flow region \\ 2: starting point of duct divergence \\ 3: starting point of pseudo-shock \\ 4: sonic point in pseudo-shock \\ 5: end point of pseudo-shock
}

\section{Introduction}

The pseudo-shock, or shock train, appears when a supersonic flow decelerates to the subsonic level. It consists of several shock waves and has a complicated flow structure. ${ }^{1)}$ When designing the supersonic wind tunnel diffusers or ramjet engines, the starting position and pressure recovery of the pseudo-shock are great concerns. ${ }^{2,3)}$ Recently, pseudo-shock has become a key phenomenon in scramjet isolators. ${ }^{4)}$ In a hot flow (e.g., the ramjet engines), heat flux in the pseudoshock is also a great concern when designing the cooling system. ${ }^{5,6)}$ Heat flux in the pseudo-shock is approximately proportional to 0.8 power of the pressure ratio.

To predict the starting position of the pseudo-shock, Kanda et al. proposed the momentum balance model. ${ }^{7,8)}$ It discusses balance between the impulse functions into/from the region containing the pseudo-shock, considering the friction and reaction force from the wall. The model is based on the conservation laws of mass, energy and momentum. It succeeded to calculate the starting positions or the lengths of pseudo-shocks under various conditions. The inflow boundary layer condition affects the position of the pseudo- 
shock, especially in a straight duct. To balance the inflow and outflow impulse functions, the momentum thickness or friction upstream of the pseudo-shock greatly affects the starting position. The thickness also affects the pressure at the end of the pseudo-shock. ${ }^{7,9)}$

In divergent ducts, the model used the linear distribution of pressure in the pseudo-shock and calculated the reaction force from the wall. The model showed some success in calculating the starting position of the pseudo-shock. ${ }^{7)}$ After confirmation and assessment, the model was applied to design a diffuser of the Rocket Based Combined Cycle (RBCC) research engine. ${ }^{10)}$ The engine operated and produced thrust as designed. ${ }^{11,12)}$ The positions of the pseudo-shock reasonably agreed with the positions designed for ejector-jet and ramjet mode operation.

The model uses linear pressure distribution in the pseudoshock. No problems occur when the model is applied to a straight duct; however, there is a problem when applied to a divergent duct. Although the RBCC research engine operated successfully, there was still a problem in accurately estimating the reaction force from the wall. The linear pressuredistribution model overestimates the length of the pseudoshock. For example, Nagata et al. used parabolic pressure distribution to determine the pseudo-shock. ${ }^{13)}$ When designing a divergent duct, the wall angle is a parameter. It is specified so that decelerated flow does not separate from the wall. From the viewpoint of designing the pseudo-shock position, the angle is a parameter. It is not specified in the linear pressure-distribution model, in which only cross-sections are specified as parameters for the duct geometry.

The supersonic core flow decelerates to the subsonic level during the pseudo-shock; meaning that it drops to below the speed of sound (i.e., sonic condition) during deceleration. In the present study, the sonic condition is applied to calculate the starting position, or length of the pseudo-shock. By modifying pressure distribution using the sonic condition, the starting position of the pseudo-shock can be accurately calculated. At the same time, the sonic-condition model reflects a duct geometrical feature of divergence angle or length. Physical models and the calculation procedure are explained first. The results calculated are then compared with our experimental results and those of other studies. Finally, the effectiveness and features of the present sonic-condition model and characteristics of the pseudo-shock are discussed.

\section{Calculation Model for Pseudo-Shock Position}

\subsection{Force balance}

Schlieren pictures or sketches show that the pseudo-shock consists of the core flow region and the peripheral lowvelocity region on the wall. ${ }^{1,13,14)}$ Figure 1 shows a schematic of the pseudo-shock in a divergent duct. To balance the force in the divergent duct flow, the sum of the inflow impulse function, $F_{1}$, and reaction force from the divergent wall, $F_{w}$, is equal to the sum of the friction force, $F_{f}$, and outflow impulse function, $F_{5}$.

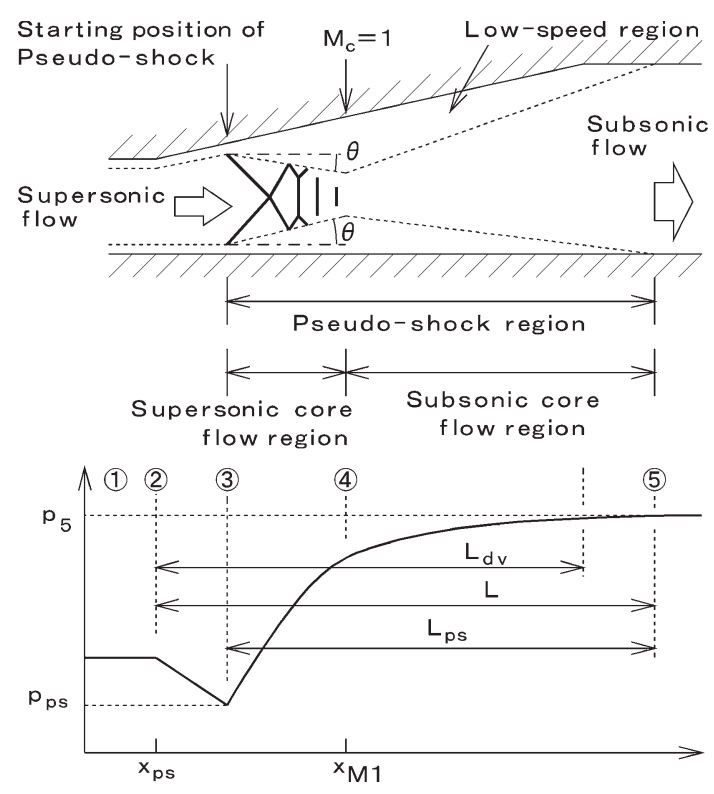

Fig. 1. Schematic diagram of pseudo-shock in a divergent duct.

$$
F_{1}+F_{w}=F_{f}+F_{5}
$$

In the pseudo-shock, the boundary layer or low-velocity region around the core flow is thick. In this region, friction becomes small or negative due to the small velocity gradient or separation. ${ }^{15-17)}$ For simplicity, pseudo-shock friction is set to 0 in the force balance calculation. This is an outline of the previous calculation model to determine the starting position of the pseudo-shock. ${ }^{7)}$ The reaction force from the wall was calculated using the mean pressure and projected cross-section. The mean pressure was an average of that at the starting position of the pseudo-shock, $p_{3}$, and that at the end position, $p_{5}$. In other words, pressure in the pseudo-shock was presumed to be distributed linearly. In the present study, the distribution is modified by taking the flow structure of the pseudo-shock into consideration.

Supersonic core flow gradually decelerates subsonic through shock waves in the pseudo-shock. In deceleration, the core flow passes by a sonic condition. In the present calculation model, the change in core flow is divided into two parts; deceleration from the supersonic condition at the starting position to the sonic condition, and further deceleration from the sonic condition to the subsonic condition at the exit of the pseudo-shock.

Generally, the impulse function, $F$, is written as

$$
F=\frac{\dot{m} \sqrt{\gamma R T_{t}}}{\sqrt{1+\frac{\gamma-1}{2} M^{2}}}\left(M+\frac{1}{\gamma M}\right)
$$

$F$ decreases as the Mach number decreases, and becomes minimum when the supersonic flow reaches the sonic condition. In a subsonic flow, $F$ increases as the Mach number decreases. In the pseudo-shock, the impulse function of the supersonic core flow decreases as the flow decelerates to sonic speed. 
The impulse function of the supersonic core flow at the starting position of the pseudo-shock is

$$
F_{3}=\dot{m}_{3} u_{3}+p_{3} A_{3}
$$

It is presumed that there is no low-velocity region at the starting position. The impulse functions of the core flow, indicated by subscript $c$, and the low-velocity region, indicated by subscript $l$, at the sonic position are written as follows, respectively:

$$
\begin{aligned}
& F_{4 c}=\dot{m}_{4 c} u_{4 c}+p_{4} A_{4 c} \\
& F_{4 l}=p_{4} A_{4 l}
\end{aligned}
$$

As explained below Eq. (1), in the low-velocity region around the core flow, the momentum is small because of the low speed. For simplicity, it is presumed the speed is 0 in the low-velocity region when calculating force balance. Some type of flow structure is needed to assist force balance in the region. However, that is out of the scope of this study and will require further research to clarify the structure.

The total impulse function at the sonic point, that is, sum of that of the core flow and that of the low-velocity region, is

$$
F_{4}=F_{4 c}+F_{4 l}
$$

Here,

$$
\begin{aligned}
& \dot{m}_{4 c}=\dot{m}_{3} \\
& A_{4}=A_{4 c}+A_{4 l} \\
& p_{4}=p_{4 c}=p_{4 l}
\end{aligned}
$$

The cross section of the supersonic core flow decreases and that of the low-velocity region increases as it nears the sonic point. At the same time, the impulse function of the core flow decreases, and that of the low-velocity region increases as the pressure and cross-section increase.

Downstream of the sonic point of the core flow, the core flow diverges. At the end of the pseudo-shock, it is presumed there is one uniform region consisting the core flow and lowvelocity region.

At the end of the pseudo-shock, the impulse function, $F_{5}$, is larger than that at the starting position of the pseudo-shock, $F_{3}$, due to the reaction force from the divergent wall.

$$
F_{5}=F_{3}+\bar{p}_{34} \Delta A_{34}+\bar{p}_{45} \Delta A_{45}
$$

The average pressure and increase in the cross section are calculated in each part, respectively.

The impulse function at the exit of the pseudo-shock is expressed in another way as

$$
F_{5}=\dot{m}_{5} u_{5}+p_{5} A_{5}
$$

\subsection{Core flow characteristic at sonic condition}

The characteristics of the supersonic core flow in the pseudo-shock are examined here. The impulse function is expressed as

$$
F=\dot{m} u+p A=p A\left(\gamma M^{2}+1\right)
$$

At the starting position of the pseudo-shock,

$$
F_{3}=p_{3} A_{3}\left(\gamma M_{3}^{2}+1\right)
$$

The core flow and low-velocity regions exist at location 4. The Mach number of the core flow is unity.

$$
\begin{aligned}
& F_{4 c}=p_{4} A_{4 c}(\gamma+1) \\
& F_{4 l}=p_{4} A_{4 l}=p_{4}\left(A_{4}-A_{4 c}\right) \\
& F_{4}=p_{4} A_{4}\left(\gamma \frac{A_{4 c}}{A_{4}}+1\right)
\end{aligned}
$$

At the same time, the impulse function at position $4, F_{4}{ }^{\prime}$, is written in another way, as sum of the inflow impulse function and reaction force from the divergent wall.

$$
\begin{aligned}
F_{4}{ }^{\prime} & =F_{3}+\left(A_{4}-A_{3}\right) \times \bar{p} \\
& \approx F_{3}+\left(A_{4}-A_{3}\right) \times \frac{1}{2}\left(p_{3}+p_{4}\right)
\end{aligned}
$$

In Eq. (17), the reaction force is calculated using the average pressures at the starting position of the pseudo-shock and at the core-flow sonic point. Since $F_{4}{ }^{\prime}=F_{4}$,

$$
\begin{aligned}
\frac{F_{4}}{F_{3}} & =1+\frac{\left(\frac{A_{4}}{A_{3}}-1\right) \times \frac{1}{2}\left(\frac{p_{4}}{p_{3}}+1\right)}{1+\gamma M_{3}^{2}} \\
& =\frac{A_{4}}{A_{3}} \cdot \frac{p_{4}}{p_{3}} \cdot \frac{1+\gamma \frac{A_{4 c}}{A_{4}}}{1+\gamma M_{3}^{2}}
\end{aligned}
$$

The mass flow rate is then expressed as

$$
\dot{m}=\rho u A=p \cdot A \cdot M \sqrt{\frac{\gamma}{R T_{t}}} \sqrt{1+\frac{\gamma-1}{2} M^{2}}
$$

Since $\dot{m}_{4}=\dot{m}_{3}, p_{4} / p_{3}$ is expressed as

$$
\frac{p_{4}}{p_{3}}=\frac{A_{3}}{A_{4 c}} M_{3} \frac{\sqrt{1+\frac{\gamma-1}{2} M_{3}^{2}}}{\sqrt{1+\frac{\gamma-1}{2}}}
$$

At position 4, the Mach number of the core flow is unity. Substituting Eq. (20) for Eq. (18), the following equation is derived.

$$
\begin{gathered}
\left(1+\gamma M_{3}{ }^{2}\right)+\frac{1}{2}\left(\frac{A_{4}}{A_{3}}-1\right) \frac{A_{3}}{A_{4 c}} M_{3}\left(\frac{\sqrt{1+\frac{\gamma-1}{2} M_{3}^{2}}}{\sqrt{1+\frac{\gamma-1}{2}}}+1\right) \\
=\frac{A_{4}}{A_{3}} \cdot \frac{A_{3}}{A_{4 c}} M_{3} \frac{\sqrt{1+\frac{\gamma-1}{2} M_{3}^{2}}}{\sqrt{1+\frac{\gamma-1}{2}}}\left\{1+\gamma \frac{A_{4 c}}{A_{4}}\right\}
\end{gathered}
$$

Here,

$$
A_{4 c} / A_{4}=\left(A_{4 c} / A_{3}\right) \cdot\left(A_{3} / A_{4}\right)
$$

When the inflow Mach number, $M_{3}$, is specified, $A_{4 c} / A_{3}$ and $A_{4} / A_{3}$ are parameters. 


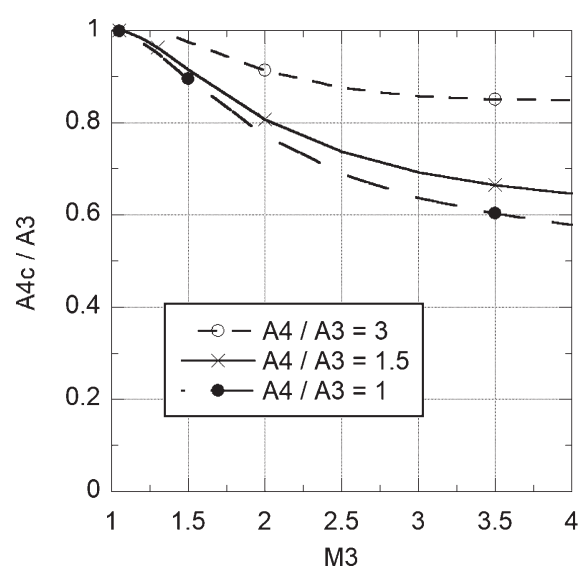

Fig. 2. Relation between the cross-section ratio, $A_{4 c} / A_{3}$, and the Mach number, $M_{3}$.

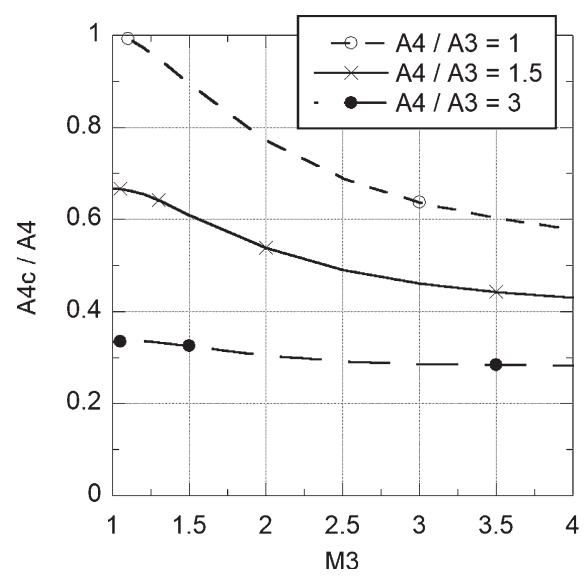

Fig. 3. Relation between the cross-section ratio, $A_{4 c} / A_{4}$, and the Mach number, $M_{3}$.

The length from the starting position of the pseudo-shock to the core-flow sonic point, $L_{M 1}$, is expressed with a crosssection ratio $A_{4 c} / A_{3}$ as below

$$
\frac{L_{M 1}}{\sqrt{A_{3}}}=\frac{1}{2}\left(1-\sqrt{\frac{A_{4 c}}{A_{3}}}\right) \times \frac{1}{\tan \theta}
$$

where, $\theta$ is a convergent angle of the supersonic core flow. When $\theta, A_{3}$ and $A_{4 c}$ are specified, $L_{M 1}$ can be calculated. At the same time, $L_{M 1}$ is calculated from the geometrical conditions of the duct $\left(A_{4} / A_{3}\right)$, using Eqs. (21) and (22). Once $\theta$ is specified, the effect of the duct divergence angle is reflected in the present model as a design parameter.

Figure 2 shows the ratio of the core-flow cross-section at the sonic point to the cross-section at the starting position of the pseudo-shock against the Mach number at the starting position of the pseudo-shock, $M_{3}$. The ratio becomes smaller as the Mach number increases, and as the ratio of $A_{4} / A_{3}$ increases. The ratio of $A_{4 c} / A_{3}$ becomes approximately $0.6-$ 0.9 in high Mach numbers. Figure 3 shows the relationship between the cross-section ratio, $A_{4 c} / A_{4}$, and Mach number at the starting position of the pseudo-shock. The core-flow cross-section ratio becomes smaller as the Mach number increases. This feature is clear when $A_{4} / A_{3}$ is small. This fea-

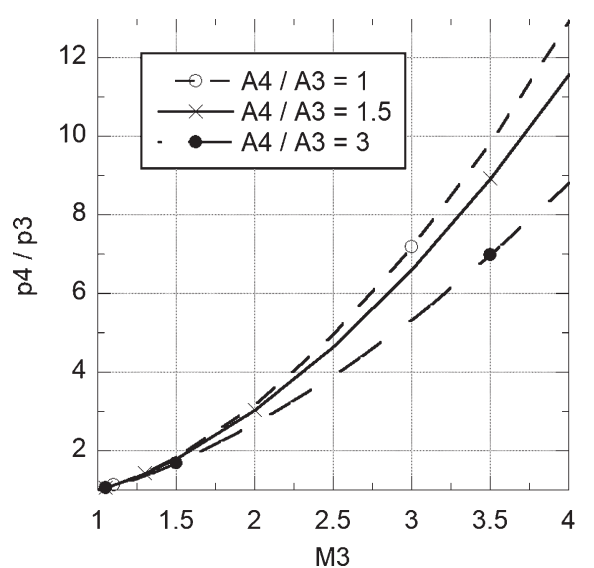

Fig. 4. Pressure ratios at the sonic point at the starting point of the pseudoshock.

ture agrees with the schlieren pictures and sketches of the supersonic core flow in the pseudo-shock. ${ }^{1,13,14)}$

Figure 4 shows the ratio of pressure at the sonic point, $p_{4}$, to that at the starting point of the pseudo-shock, $p_{3}$, against the Mach number, $M_{3}$. The ratio increases as the Mach number increases. The quick pressure increase near the starting position of the pseudo-shock is caused by this large pressure increase up to the sonic point.

\subsection{Friction}

No friction is presumed in the pseudo-shock, as was done in the previous model. ${ }^{7)}$ Friction works upstream of the pseudo-shock in the present model. The turbulent skin friction coefficient is calculated using the formula of White. ${ }^{18)}$

$$
\begin{aligned}
& c_{f} \approx \frac{0.455}{S^{2} \ln ^{2}\left(\frac{0.06}{S} R e_{x} \frac{\mu_{e}}{\mu_{w}} \sqrt{\frac{T_{e}}{T_{w}}}\right)} \\
& S=\frac{\sqrt{\frac{T_{a w}}{T_{e}}-1}}{\sin ^{-1} A+\sin ^{-1} B} \\
& A=\frac{2 a^{2}-b}{\left(b^{2}+4 a^{2}\right)^{\frac{1}{2}}}, \quad B=\frac{b}{\left(b^{2}+4 a^{2}\right)^{\frac{1}{2}}} \\
& a=\left(\frac{\gamma-1}{2} M_{e}{ }^{2} \frac{T_{e}}{T_{w}}\right)^{\frac{1}{2}}, \quad b=\frac{T_{a w}}{T_{w}}-1
\end{aligned}
$$

The wall is presumed to be adiabatic. The recovery factor is set to 1 .

\subsection{Calculation procedure}

Inflow and outflow boundary conditions, that is, the inflow Mach number and the pressure ratio at the duct exit, are specified. The starting position of the pseudo-shock is determined to satisfy the force balance of Eq. (1). First, the starting position of the pseudo-shock is presumed. Once the inflow impulse function into the pseudo-shock, $F_{3}$, is specified, parameters at the sonic condition are determined. Then, the force balance is calculated at the end point of the pseudo-shock downstream of the sonic point. Finally, the 
starting position of the pseudo-shock is renewed to obtain the actual force balance.

In some experiments, there was a straight duct downstream of the divergent duct, and wall pressure continued to increase gradually in the straight duct. In the present calculation, the end point of the pseudo-shock is at the end of the divergent duct. Friction downstream in the straight duct can affect the starting position of the pseudo-shock. However its effect on the position is much smaller than the effect of the reaction force from the divergent wall. Therefore, the end of the pseudo-shock is set at the end of the divergent duct, and the starting position of the pseudo-shock is calculated. When comparing the length of the pseudo-shock, $L_{p s}$, between the experiments and calculations, $L_{p s}$ is calculated using the distance of the starting position of the pseudo-shock, $x_{p s}$, and the duct length from the starting position of the divergent duct to the end of the duct, including the downstream straight duct in some cases, $L$.

$$
x_{p s} / L=1-L_{p s} / L
$$

$L_{M 1}$ is calculated applying $A_{4} / A_{3}$ of the duct geometrical conditions, using Eqs. (21) and (22). On the other hand, according to pictures and sketches, ${ }^{1,13,14)}$ the convergent angle of the core flow is small. In this study, the reference convergence angle of the core flow, $\theta$, is set to $2 \mathrm{deg}$. With $\theta$, another $L_{M 1}$ is calculated using Eq. (23) to calculate the convergence.

Herein, the core-flow convergence angle presumed is compared to the growth rate of the compressible turbulent shear layer following the experimental equation of Papamoschou and Roshko. ${ }^{19)}$

$$
d \delta / d x=(d \delta / d x)_{0} \cdot f\left(\bar{M}_{c}\right)
$$

When fluids in the core flow and low-velocity region are the same, the growth rate of the incompressible shear layer is expressed as follows. ${ }^{19)}$

$$
\left(\frac{d \delta}{d x}\right)_{0}=\frac{0.14}{1+\left(M_{l} / M_{c}\right)} \times\left\{1-\left(M_{l} / M_{c}\right)-A+B\right\}
$$

Here,

$$
\begin{aligned}
A & =\frac{M_{l}}{M_{c}} \sqrt{\frac{1+\{1+(\gamma-1) / 2\} M_{l}^{2}}{1+\{1+(\gamma-1) / 2\} M_{c}^{2}}}, \\
B & =\sqrt{\frac{1+\{1+(\gamma-1) / 2\} M_{l}^{2}}{1+\{1+(\gamma-1) / 2\} M_{c}^{2}}}
\end{aligned}
$$

The effect of compressibility, $f$, can be expressed as a function of the convective Mach number, $\bar{M}$. The empirical equation of Dimotakis ${ }^{20)}$ is used to predict $f$.

$$
f(\bar{M})=\left(1-f^{\prime}\right) \exp \left(-3 \bar{M}^{2}\right)+f^{\prime}
$$

$f^{\prime}$ is approximately 0.2 . The definitions of the convective Mach number and convective velocity, $\bar{u}$, are as follows, respectively.

$$
\begin{aligned}
\bar{M}_{c} & =\left(u_{c}-\bar{u}\right) / a_{c} \\
\frac{\bar{u}}{u_{c}} & =\frac{1+\left(u_{l} / u_{c}\right) \sqrt{\rho_{l} / \rho_{c}}}{1+\sqrt{\rho_{l} / \rho_{c}}}
\end{aligned}
$$

When the core flow Mach number, $M_{3}$, is 3 and that of the low-velocity region, $M_{l}$, is $0, d \delta / d x=0.049$ and $\theta=2.8 \mathrm{deg}$. When $M_{3}$ is 4 and $M_{l}=0, d \delta / d x=0.043$ and $\theta=2.4 \mathrm{deg}$. These results are close to the presumed convergence angle of the present model. The accuracy of $L_{p s}$ calculated using the present model will improve when using a suitable convergence angle model.

\subsection{Linear pressure-distribution model and normal shock model}

The effect of pressure distribution on the starting position of the pseudo-shock is compared with other models. One is the previous calculation model (Linear). ${ }^{7)}$ The mean pressure in the pseudo-shock is calculated using the pressure at the starting position and that at the end of the pseudo-shock. Therefore, linear pressure distribution is presumed in the pseudo-shock. The starting position of the pseudo-shock is calculated using the force balance, as well as the present model. In another model, the normal shock relation is used (N.S.), where the exit pressure is used for the downstream boundary condition. Upstream and downstream of the shock, the isentropic condition is used. Although there are other calculation methods for determining the starting position of the pseudo-shock, none of them showed good agreements, ${ }^{7)}$ so they are not compared here.

\section{Experimental Apparatus}

Experiments were conducted in a Mach 2 blow-down supersonic wind tunnel at Chubu University. Figure 5 shows the wall configuration and positions where wall pressures were measured. The core flow Mach number is 1.88 . The nozzle exit is open to ambient air and is $40 \mathrm{~mm} \times 40 \mathrm{~mm}$ square. The throat height is $24.2 \mathrm{~mm} \times 40 \mathrm{~mm}$. The $99 \%$ velocity boundary layer thickness is $3 \mathrm{~mm}$. The maximum air temperature is approximately $290 \mathrm{~K}$, and the maximum pres-

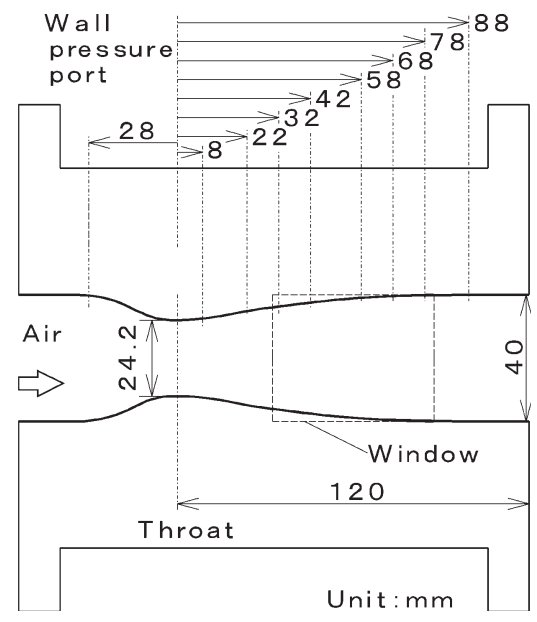

Fig. 5. Schlieren diagram of Mach 2 supersonic wind tunnel nozzle. 
sure is $0.9 \mathrm{MPa}$. Pressure is measured using diaphragm-type SMC ZSE40AF $100 \mathrm{kPa}$ sensors and ISE40A $1 \mathrm{MPa}$ sensors. The accuracy of the sensors is $\pm 1 \%$. The sampling rate is $10 \mathrm{kHz}$, and instantaneous pressure is measured. The diameters of the wall pressure taps are $1 \mathrm{~mm}$. Each pressure tap is connected to a sensor using a $0.5 \mathrm{~m} \mathrm{TYGON}^{\circledR}$ tube.

The pseudo-shock was created in the nozzle by reducing the reservoir pressure. The total pressure gradually decreased during the $15 \mathrm{~s}$ test period from $0.9 \mathrm{MPa}$ to $0.3 \mathrm{MPa}$, and the wall pressure was measured every $0.1 \mathrm{~s}$.

\section{Results and Discussion}

\subsection{Wall pressure distribution}

Figure 6 shows the wall pressure ratios measured in the Mach 2 nozzle and ratios in the study by Nagata et al. ${ }^{13)} x$ is the distance from the starting position of the divergent duct. The measured pressure is normalized by the inflowair total pressure. The pressure ratios calculated are plotted for comparison.

The measurements show that the wall pressure ratios increase quickly after the starting position of the pseudo-shock, and then gradually increase. The ratios calculated using the

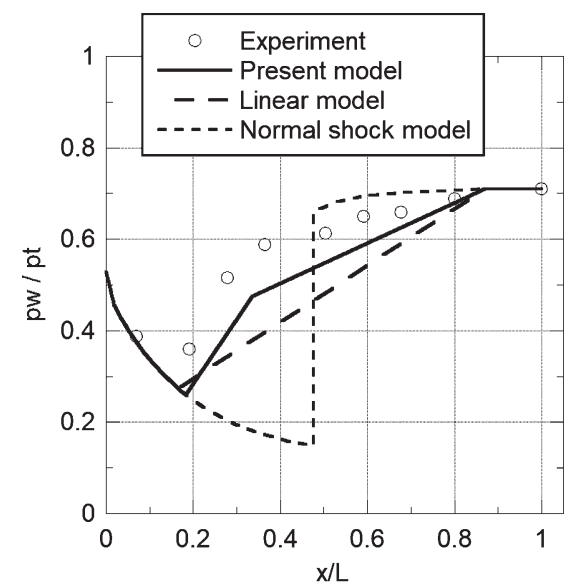

(a)

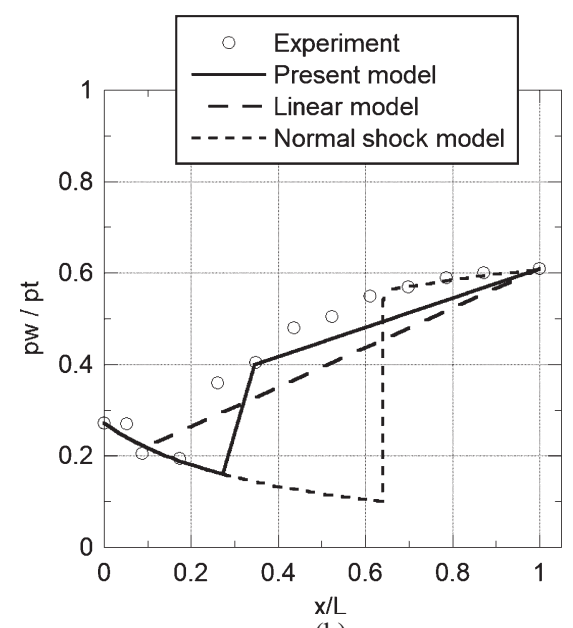

(b)

Fig. 6. Experimental and calculated distributions of wall pressure ratio of Mach 2 nozzle test (a) and test by Nagata et al. (b). ${ }^{13)}$ In (a), $p_{e} / p_{t 1}=0.71$. In (b), inflow Mach is 1.5 , and $p_{e} / p_{t 1}=0.61$. present model show reasonable agreement with the experimental ratios. The quick increase in pressure near the starting position of the pseudo-shock is simulated. The improvement is attained using the sonic condition.

There are still discrepancies between the experimental pressure and that calculated. One cause is the convergence angle of the core flow mentioned previously. Another is assuming that there is no velocity in the low-velocity region, which affects the force balance. Further study of these subjects is necessary.

The starting positions of the linear model are upstream of the experimental positions. In the linear model, the wall pressure distribution is lower than the actual one; therefore, the region of the pseudo-shock becomes longer. Under the Mach 2 test conditions, the difference in the starting positions between the present model and the linear model is small. In the Mach 2 nozzle, the pressure and cross-section change largely near the throat because the nozzle is contoured. Therefore, the upstream nozzle has a large effect on the reaction force, and the difference in the starting position is small between the models.

In the test conditions of Nagata et al., the cross-section of the divergent duct increases linearly. So the starting position locates far upstream of the experimental position. The linear model calculates starting position with some accuracy under these flow conditions. However, the model cannot calculate wall pressure distribution or length of the pseudo-shock under some conditions. This shortage will be discussed in the next section.

When position accuracy is required, or when a cooling system with the pseudo-shock is designed, the present model is better than the linear model. The starting positions of the normal shock model are located downstream of the actual ones, and the length of the pseudo-shock is calculated shorter.

\subsection{Comparison between experimental and calculated} lengths of the pseudo-shock

Figure 7 shows comparisons between experimental and calculated results for the length ratio of the pseudo-shock to the duct, $L_{p s} / L$. Experimental conditions and results from the study conducted by Nagata et al., ${ }^{13)}$ Penzin $^{21)}$ and Ikui et al. $^{22)}$ are used for comparison. The test conditions are listed in Table 1. The results calculated using the linear pressure-distribution model and those using the normal shock model are also plotted.

In Fig. 7(a), the results calculated by Nagata et al. under experimental conditions are lower than our experimental results. Under these conditions, the pressure ratio at the duct exit is 0.238 compared to the total pressure of the inflow air, which is lower than the results reported by others. The pseudo-shock may not be sufficiently constructed under this exit pressure ratio, and the calculated pressure level becomes higher than the experimental pressure. This higher pressure causes the length of the pseudo-shock calculated to be shorter.

When the linear model is applied to the conditions used by Penzin, a pseudo-shock length that is longer than the geometrically determined length is necessary to attain force balance under some conditions. They are indicated as $L_{p s} / L>1$ in 


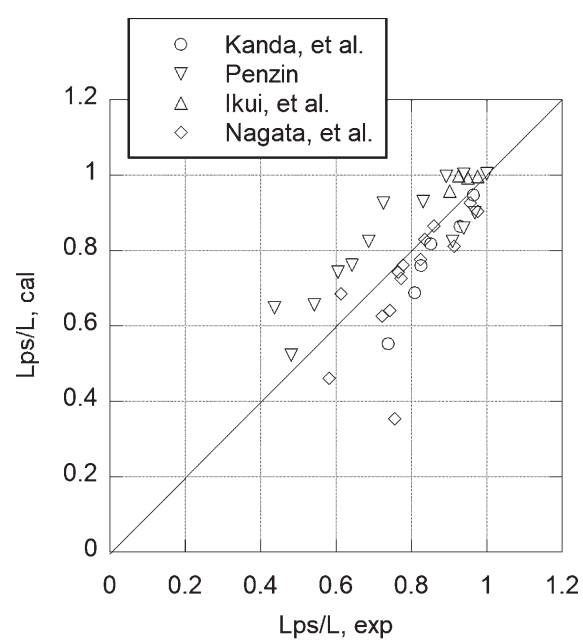

(a)

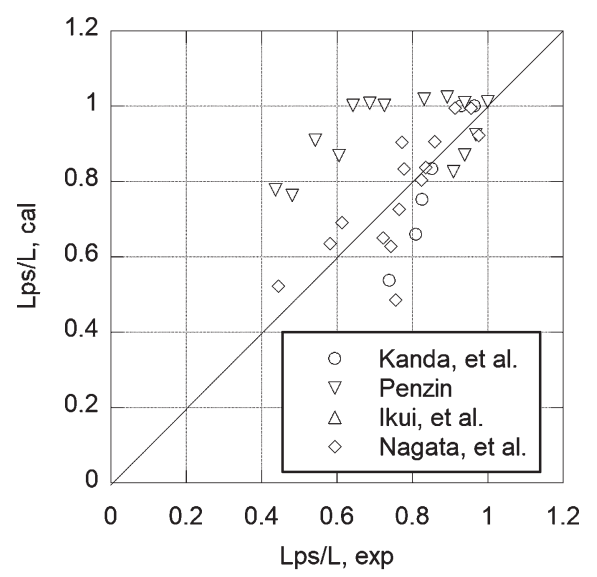

(b)

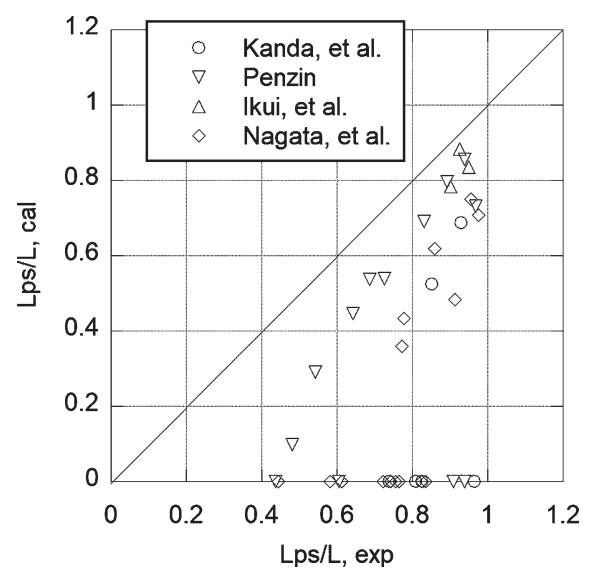

(c)

Fig. 7. Comparison between the calculated and experimental lengths of pseudo-shock.

(a) Present model, (b) Linear interpolation model, and (c) Normal shock model.

the figure. When the linear model is used, the pressure calculated is lower than that in the experiments, especially around the starting position of the pseudo-shock. This lower pressure causes $L_{p s} / L>1$ failure. This inaccuracy is improved in the present model. When the normal shock model is applied, there is no solution in some cases. They are indicated as $L_{p s} / L=0$.
Table 1. Test conditions.

\begin{tabular}{lcccc}
\hline & $\begin{array}{c}\text { Inflow Mach } \\
\text { No. }\end{array}$ & $p_{5} / p_{t 1}$ & $\begin{array}{c}\text { Wall half angle, } \\
\text { deg }\end{array}$ & $A_{5} / A_{1}$ \\
\hline Kanda et al. & 1 & $0.60-0.82$ & Contoured nozzle & 1.67 \\
Penzin & $2.6,3.2,3.8$ & $0.11-0.34$ & $0.5,2$ & $1.3,1.67,2.5$ \\
Ikui et al. & 1.8 & $0.55-0.67$ & $2.2,4.2$ & $2.56,3.98$ \\
Nagata et al. & $1.5,2.5$ & $0.23-0.7$ & $2.5,5,6.7$ & 2 \\
\hline
\end{tabular}

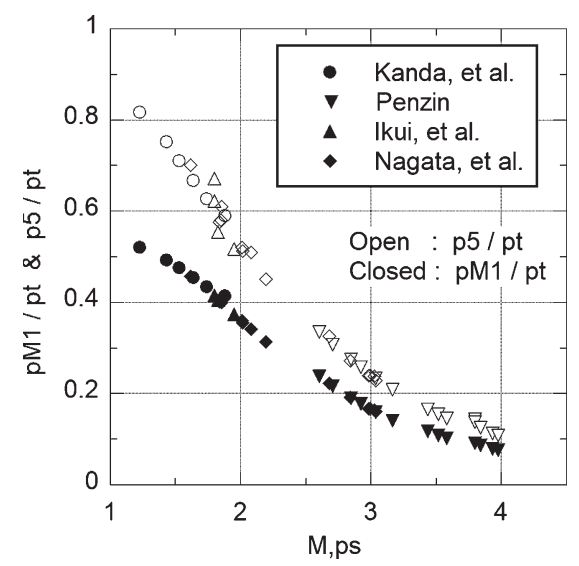

Fig. 8. Ratios of sonic point pressure and duct exit to inflow total pressure.

As can be seen in Fig. 7(a), lengths calculated with the present, sonic-condition model show reasonable agreement with those obtained during experiments. By comparing Fig. 7(a) and (b), accuracy in calculating the starting position of the pseudo-shock is improved and it is shown that the model can be applied to larger flow conditions; however, the calculation process becomes more complicated.

Since the effectiveness of the present sonic condition model is made clear, the sonic condition will work for determining the actual pseudo-shock. In the following, features of the pseudo-shock are examined based on the present model calculation results. Figure 8 shows ratios of the pressure at the sonic point calculated with the present model to total pressure at the duct entrance against the Mach number at the starting position of the pseudo-shock. Ratios of the pressure at the exit to the total pressure are also plotted. The ratios decrease as the Mach number increases. Figure 9 shows ratios of pressure at the sonic point to that at the duct exit against the Mach number at the starting position of the pseudo-shock. An increase in pressure approximately 0.7 is achieved by the sonic point in the pseudo-shock.

Next, the position of the sonic point is examined. According to the model calculation results, pressure will increase quickly by the sonic point of the core flow. Figure 10 shows a comparison between lengths of the quick-pressure-increase region in the experiments, $L_{q}$, and lengths from the starting position to the sonic point in the calculation, $L_{M 1}$. The lengths are normalized by height at the duct entrance, $h_{1}$. Figure 10 shows some relation between the experimental results and those calculated. Therefore, presuming there is a relation between $L_{q}$ and $L_{M 1}, L_{q}$ is compared to the results calculated for $L_{M 1}$. Figure 11 shows the length ratios of 


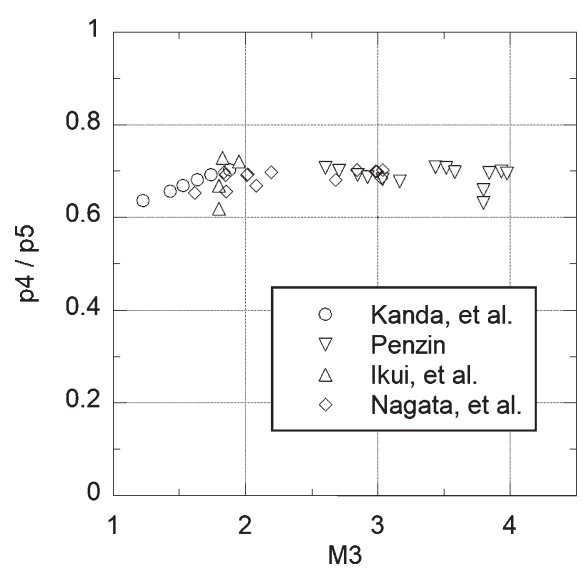

Fig. 9. Ratios of sonic point pressure to that at the duct exit.

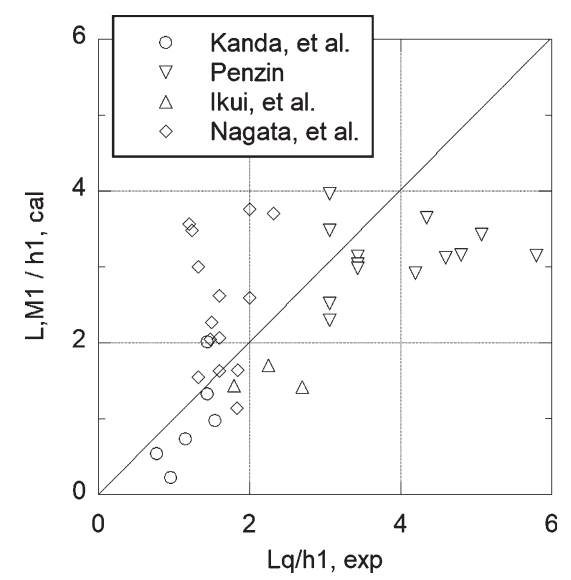

Fig. 10. Relation between the length ratio of quick-pressure-increase region and that from the starting position of the pseudo-shock to the sonic point.

$L_{M 1} / h_{1}$ against the Mach number at the starting position. The length ratio increases as the Mach number increases until Mach 3. In the high Mach numbers, the length ratio is around 3.5. In the experiments, the length of the quick-pressureincrease region looks to become longer as the inflow Mach number increases. ${ }^{13,21)}$ The results of Fig. 11 agree with our experimental observations.

\section{Conclusion}

A new model is constructed for calculating the starting position of the pseudo-shock or shock train in divergent ducts. The model is based on the conservations of mass, energy and momentum into/from the pseudo-shock region, as well as a previous model. The present model further uses the sonic condition in the core flow of the pseudo-shock. In the model, the divergent angle of the nozzles becomes a design parameter, as does the cross-section.

The results calculated with the present sonic-condition model show reasonable agreement with the experimental results. Therefore, the model succeeded to improve accuracy in calculating the starting position and pressure distribution of the pseudo-shock. The pressure at the sonic point rises up to 0.7 of that at the duct exit. The length of the quick pres-

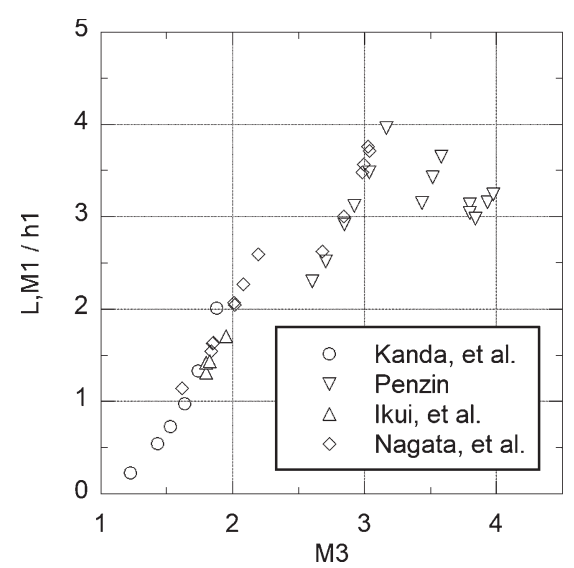

Fig. 11. Length ratio from starting point to sonic point against the Mach number at the starting point of the pseudo-shock.

sure-increase region becomes longer against the Mach number at the starting position of the pseudo-shock.

\section{Acknowledgments}

The authors wish to thank Mr. Tatsuro Asano and Mr. Yasutaka Okuma, undergraduate students of Chubu University, for discussions with us.

This study is supported by JSPS KAKENHI Grant Number $18 \mathrm{~K} 03957$.

\section{References}

1) Matsuo, K., Miyazato, Y., and Kim, H.-D.: Shock Train and PseudoShock Phenomena in Internal Gas Flows, Progr. Aerospace Sci., 35 (1999), pp. 33-100. doi: 10.1016/S0376-0421(98)00011-6

2) Geerts, J. S. and Yu, K. H.: Shock Train/Boundary-Layer Interaction in Rectangular Isolators, AIAA J., 54, 11 (2016), pp. 3450-3464. doi: 10.2514/1.J054917

3) Vanstone, L., Lingren, J., and Clements, N. T.: Simple Physics-Based Model for the Prediction of Shock-Train Location, J. Propul. Power, 34, 6 (2018), pp. 1428-1441. doi: 10.2514/1.B37031

4) Huang, H., Tan, H., Sun, S., and Wang, A.: Behavior of Shock Train in Curved Isolators with Complex Background Waves, AIAA J., 56, 1 (2018), pp. 329-341. doi: 10.2514/1.J056166

5) Back, L. H., Cuffel, R. F., and Massier, P. F.: Flow and Heat Transfer Measurements along a Cooled Supersonic Diffuser, AIAA J., 22, 6 (1984), pp. 777-780. doi: 10.2514/3.48517

6) Kato, K. and Kanda, T.: Experimental Study of Heat Flux in the Pseudo-Shock Region, Trans. Jpn. Soc. Aeronaut. Space Sci., 59 (2016), pp. 305-309. doi: 10.2322/tjsass.59.305

7) Kanda, T. and Tani, K.: Momentum Balance Model of Flow Field with Pseudo-Shock, JAXA Report, JAXA-RR-06-037E, March 2007, or AIAA Paper AIAA 2005-1045, Jan. 2005. doi: 10.2514/6.2005-1045

8) Kanda, T.: Prediction of Pseudo-Shock Position in a Long Duct Using the Momentum Balance Model, Trans. Jpn. Soc. Aeronaut. Space Sci., 55 (2012), pp. 199-201. doi: 10.2322/tjsass.55.199

9) McLafferty, G.: Theoretical Pressure Recovery through a Normal Shock in a Duct with Initial Boundary Layer, J. Aeronaut. Sci., 20, 3 (1953), pp. 169-174. doi: 10.2514/8.2582

10) Kanda, T., Tomioka, S., Ueda, S., Tani, K., and Wakamatsu, Y.: Design of Sub-Scale Rocket-Ramjet Combined Cycle Engine Model, 56th IAC Paper, IAC-05-C4.5.03, Fukuoka, Japan, 2005, or JAXA Technical Report, RR-06-009E, 2009. doi: 10.2514/6.IAC-05-C4.5.03

11) Tomioka, S., Takegoshi, M., Kudo, K., Kato, K., Hasegawa, S., and Kobayashi, K.: Performance of a Rocket-Ramjet Combined-Cycle Engine Model in Ejector Mode Operation, AIAA Paper 2008-2618, Apr. 
2008. doi: $10.2514 / 6.2008-2618$

12) Tani, K., Izumikawa, M., Saito, T., Ono, F., and Murakami, A.: Ram and Ejector-Jet Mode Experiments of the Combined Cycle Engine in Mach 4 Flight Conditions, AIAA Paper 2008-0103, Jan. 2008. doi: 10.2514/6.2008-103

13) Nagata, T.: Prediction of Location of Pseudo-Shock in Divergent Duct, Master's Thesis, Tokyo Institute of Technology, Feb. 2009 (in Japanese). or Nagata, T., Tani, K., Kudo, K., Murakami, A., Tomioka, S., Tsuru, T., and Yamasaki, H., Proceedings of the 2009 Annual Meeting and the 10th Symposium on Propulsion System for Reusable Launch Vehicles, Northern Branch of JSASS, March, 2009, G-3, pp. 129-134 (in Japanese).

14) Chen, C. P., Sajben, M., and Kroutil, J. C.: Shock-Wave Oscillations in a Transonic Diffuser Flow, AIAA J., 17, 16 (1979), pp. 1076-1083. doi: $10.2514 / 3.61280$

15) Waltrup, P. J. and Cameron, J. M.: Wall Shear and Boundary-Layer Measurement in Shock Separated Flow, AIAA J., 12, 6 (1974), pp. 878-880. doi: 10.2514/3.49373

16) Ostras, V. N. and Penzin, V. I.: Experimental Study of Friction in a Channel with a Pseudoshock, Fluid Mech. Soviet Res., 4, 6 (1975), pp. 32-38.

17) Carroll, B. F. and Dutton, J. C.: Characteristics of Multiple Shock
Wave/Turbulent Boundary-Layer Interactions in Rectangular Ducts, J. Propul. Power, 6, 2 (1990), pp. 186-193. doi: 10.2514/3.23243

18) White, F. M.: Viscous Fluid Flow, McGraw-Hill, New York, 1974, pp. 632-644.

19) Papamoschou, D. and Roshko, A.: The Compressible Turbulent Shear Layer: An Experimental Study, J. Fluid Mech., 197 (1988), pp. 453-477.

20) Dimotakis, P.: Turbulent Free Shear Layer Mixing and Combustion, Proceedings of the 9th International Symposium on Air Breathing Engines, AIAA, Washington, DC, 1989, pp. 58-79.

21) Penzin, V. I.: Deceleration of Supersonic Flows in Smoothly Diverging-Area Rectangular Ducts, Scramjet Propulsion, Curran, E. T. and Murthy, S. N. B. (eds.), Vol. 189, Progress in Astronautics and Aeronautics, AIAA, Reston, VA, 2000, pp. 321-337. doi: 10.2514/ 5.9781600866609.0223.0367

22) Ikui, T., Matsuo, K., Mochiduki, H., and Somekawa, K.: Characteristics of Pseudo-Shock in a Divergent Duct, J. Jpn. Soc. Mech. Eng., 45, 393 (1979), pp. 611-617 (in Japanese).

Toshinori Watanabe Associate Editor 